Article

\title{
Mosquito (Diptera: Culicidae) Habitat Surveillance by Android Mobile Devices in Guangzhou, China
}

\author{
Tai-Ping Wu ${ }^{1}$, Jun-Hua Tian ${ }^{1}$, Rui-De Xue ${ }^{2, *}$, Yi-Liang Fang ${ }^{3}$ and Ai-Hua Zheng ${ }^{4}$ \\ 1 Wuhan Centers for Disease Prevention \& Control, Disinfection and Vector Control Section, Wuhan 430015, \\ China; celerwu@hotmail.com (T.-P.W.); tianjunhua1980@163.com (J.-H.T.) \\ 2 Anastasia Mosquito Control District of St. Johns County, 120 EOC Drive, St. Augustine, FL 32092, USA \\ 3 Fujian International Travel Healthcare Center, Fuzhou 350001, China; Anew627@163.com \\ 4 The State Key Laboratory of Integrated Management of Pest Insects and Rodents, Institute of Zoology, \\ Chinese Academy of Sciences, Beijing 100101, China; zhengaihua@ioz.ac.cn \\ * Correspondence: xueamcd@gmail.com; Tel.: +1-904-471-3107; Fax: +1-904-471-3189
}

Academic Editor: Brian Forschler

Received: 23 June 2016; Accepted: 2 December 2016; Published: 17 December 2016

\begin{abstract}
In 2014, Guangzhou City, South China, suffered from its worst outbreak of dengue fever in decades. Larval mosquito habitat surveillance was carried out by using android mobile devices in four study sites in May 2015. The habitats with larval mosquitoes were recorded as photo waypoints in OruxMaps or in videos. The total number of potential mosquito habitats was 342, of which $166(49 \%)$ were found to have mosquito larvae or pupae. Small containers were the most abundant potential habitats, accounting for $26 \%$ of the total number. More mosquito larvae and pupae, were found in small containers than in other objects holding water, for example, potted or hydroponic plants $(p<0.05)$. Mosquito larvae were collected from all plastic road barriers, used tires, and underground water. Aedes albopictus larvae were found from small and large containers, stumps, among others. The overall route index (RI) was 11.3, which was 14.2 times higher than the grade $\mathrm{C}$ criteria of the National Patriotic Health Campaign Committee (NPHCC), China. The higher RIs were found from the bird and flower markets, schools, and underground parking lots. The results indicated that Android mobile devices are a convenient and useful tool for surveillance of mosquito habitats, and the enhancement of source reduction may benefit the prevention and control of dengue vector mosquitoes.
\end{abstract}

Keywords: mosquito habitat; Aedes albopictus; mosquito surveillance; android mobile device; OruxMaps

\section{Introduction}

Dengue is predominantly an urban disease that is vectored by container-inhabiting Aedes mosquitoes [1,2] Guangzhou, the capital city of Guangdong province in south China, has experienced outbreaks of dengue fever for many years [3], and Aedes albopictus Skuse has been confirmed as the dengue vector [4]. The most serious outbreak of dengue fever in Guangzhou was from June to December 2014, with a report of 37,331 cases (Emergency Management Office of the People's Government of Guangdong Province). During the peak of the dengue epidemic in Guangzhou in 2014, a large-scale ground application of adulticiding was conducted, but the source reduction and larval control was neglected.

Larval mosquito surveillance involves sampling a wide range of aquatic habitats for the presence of immature mosquitoes with a dipper or plastic pipette [2,5]. The spatial distribution and abundance of immature mosquito habitats [6] have been usually recorded and analyzed by papers, pictures, Microsoft Excel program, computer models [2], Vector Control Management System databases, 
and MapVision [7]. Android mobile devices and mobile cell phones are popular tools [8,9], but these tools have not been widely used for surveillance of mosquito habitats.

The objectives of this study were to: (1) determine the distribution and abundance of immature mosquito habitats at the site of the previous dengue outbreak and (2) determine the potential application of the popular Android mobile devices for surveillance of mosquito habitats.

\section{Materials and Methods}

Four sites (Sanyu Road in Yuexiu District, Tangjing Street in Baiyun District, Gangwan Road in Huangpu District, and Fangcun Road in Liwan District, Table 1) in Guangzhou City were selected for the study on the habitat surveillance and inspection route mapping (Figure 1). The last three sites formed a triangle with the first site in the center (Figure 1). The first indigenous dengue case was reported from Tangjing Street in Baiyun District, Guangzhou City, in 2015.

Table 1. Length of inspection route and elapsed time in the study sites in Guangzhou, China.

\begin{tabular}{ccc}
\hline Inspecting Site & Length of Route $\mathbf{( k m )}$ & Elapsed Time (h) \\
\hline Sanyu road, Yuexiu District & 6.3 & 3.9 \\
Tangjing street, Baiyun D. & 3.3 & 2.8 \\
Gangwan road, Huangpu D. & 2.2 & 1.6 \\
Fangcun, Liwan D. & 2.8 & 2.1 \\
Total & 14.6 & 10.3 \\
\hline
\end{tabular}

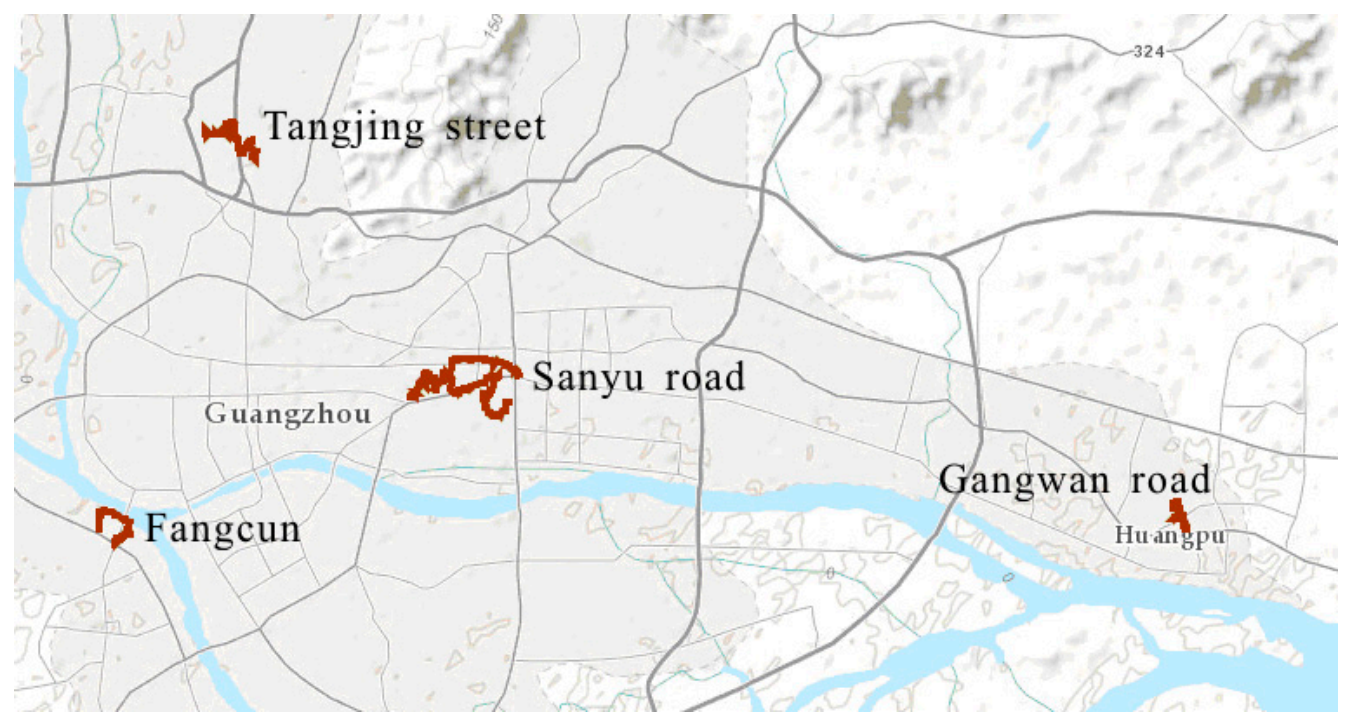

Figure 1. Four study sites (orange lines) in Guangzhou city.

Android mobile devices are very popular in China. Cameras on devices and apps, such as AutoNavi Navigation and Baidu Maps, have been facilitated for immature mosquito surveillance [8]. OruxMaps was a free map-viewing app developed by Jose Vazquez for Android mobile devices that could record routes of inspection $[9,10]$.

A Samsung Galaxy Table 3 (Samsung Electronics, Suwon, South Korea) and OruxMaps, AutoNavi Navigation (AutoNavi Holdings, Beijing, China), and Baidu Maps (Baidu, Beijing, China) were used in this study. Before surveillance, the study sites were checked in Baidu Maps and the inspection routes were planned according to the availability of premise types. Some points on the route were collected in AutoNavi Navigation. Potential habitats were categorized as small containers (less than $37 \mathrm{~cm}$ in diameter), large containers (equal to or larger than $37 \mathrm{~cm}$ in diameter), stumps (bamboo stumps or the rooted remains of metal tubes), artificial objects (such as leather shoes, toys, etc.), 
cisterns, puddles, storm drains, shallow water $(2-8 \mathrm{~cm}$ depth), depressions of tree roots, plastic road barriers, potted or hydroponic plants, fallen leaves, ditches, used tires, and subterranean pits and chambers [11]. Searches for stagnant water were conducted mainly outdoors and tracked in the log of OruxMaps with GPS. Before inspection of a new premises, a picture of the name or feature of the premises was taken. The habitats with immature mosquitoes were recorded as photo waypoints or recorded in videos. Pictures of the habitats that were negative for mosquito larvae and pupae were taken by the Android mobile device for further identification of the habitats. Auxiliary information of mosquito habitats was recorded quickly, and the immature mosquitoes were sampled with a dipper or plastic pipette [2,5] and sent to Fujian International Travel Healthcare Center in Fuzhou for species identification by morphological characters.

Track logs in a GPS Exchange Format (gpx) file contained pictures and videos that were exported to a computer. The track logs (Figure 2) appeared as polylines and were analyzed by Global Mapper 16 [12]. The vertex lists were exported to an Excel workbook to calculate the length and elapsed time of the segment in each premises. The route lengths of inspections in underground parking lots and metros near the entrance were gauged by visual estimation due to a lack of GPS signal in these locations. When an obvious error occurred in a track log between several vertexes, the length was revised by measuring the distance using the measuring tool of Global Mapper.

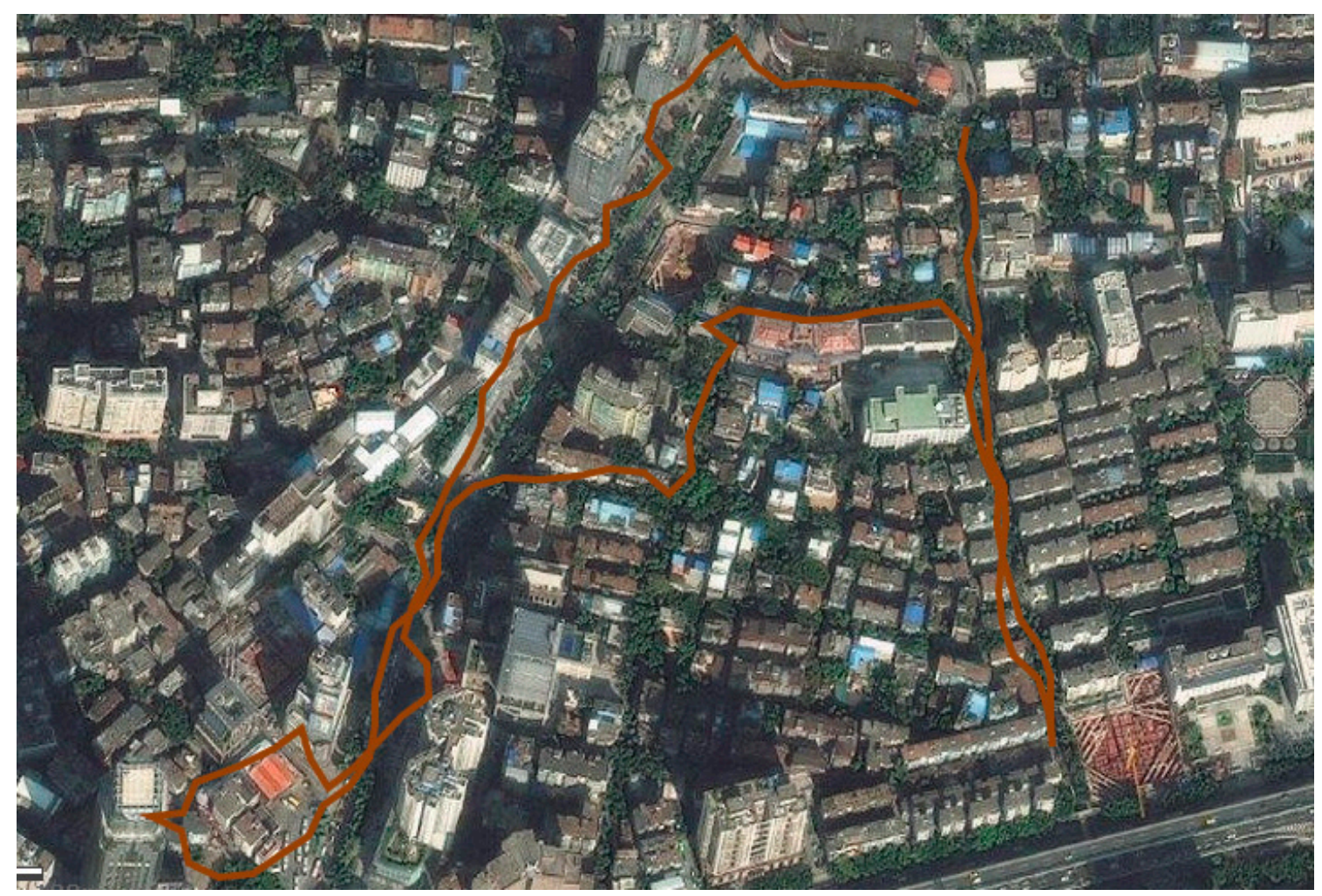

Figure 2. A part of the inspection route of the study site of Sanyu Road, Guangzhou City. The orange lines were inspection routes on an Android mobile device.

The infestation levels were estimated by using the container index (CI, percentage of water-holding containers infested with larvae or pupae); the route index (RI, the number of positive habitats per kilometer of inspection route); and the time index (TI, the number of positive habitats per hour of inspection time).

The criteria for vector density control (Mosquito: GB/T 27771-2011) was established by the Chinese National Sanitary City Standard of the National Patriotic Health Campaign Committee (NPHCC), 1 January 2015. Container Index (CI) was abandoned [2] and route index (RI) — the number 
of positive habitats on a designated route per $1000 \mathrm{~m}$-was used for the justification of reducing mosquito-positive habitats. The RI was divided into grade A (equal to or less than 0.1), grade B (equal to or less than 0.5 ), and grade $\mathrm{C}$ (equal to or less than 0.8 ). The length of inspection varied with each inspector [10].

Data was analyzed by chi-square $\left(\mathrm{X}^{2}\right)$ and Fisher's exact tests using SPSS 16.0 for Windows (SPSS, Chicago, IL, USA). Premises types were sorted into five broad categories (Table 2): road and around, residential area, underground, farmer's markets, and other resources. Proportions of all habitat types between the five broad categories of premises types cannot be computed by Fisher's exact tests with SPSS. Some habitat types and broad categories of premises types were truncated to facilitate statistical analysis.

Table 2. Number of inspection route segments, number of mosquito-positive habitats, and larval indices in Guangzhou, by premises type.

\begin{tabular}{|c|c|c|c|c|c|}
\hline Premise Type & $\begin{array}{l}\text { Number of } \\
\text { Segments }\end{array}$ & $\begin{array}{l}\text { Number of Mosquito } \\
\text { Positive Habitats }\end{array}$ & CI (\%) & RI & TI \\
\hline \multicolumn{6}{|l|}{ Road and around } \\
\hline Roadside & 27 & 44 & 42 & 4.2 & 8.0 \\
\hline Wasteland & 2 & 6 & 50 & 36.5 & 26.8 \\
\hline Subtotal & 29 & 50 & 43 & 4.7 & 8.7 \\
\hline \multicolumn{6}{|l|}{ Residential area } \\
\hline Old residential area & 7 & 17 & 57 & 31.4 & 22.8 \\
\hline Property management residential area & 1 & 1 & 50 & 30.3 & 3.1 \\
\hline Village in city & 2 & 10 & 50 & 22.7 & 35.3 \\
\hline Subtotal & 9 & 28 & 54 & 27.6 & 20.8 \\
\hline \multicolumn{6}{|l|}{ Underground } \\
\hline Underground parking lot & 3 & 8 & 100 & 70.9 & 21.3 \\
\hline Metro near the entrance & 2 & 2 & 100 & 50.0 & 30.0 \\
\hline Subtotal & 5 & 10 & 100 & 65.4 & 22.6 \\
\hline Farmer's market & 3 & 0 & 0 & 0.0 & 0.0 \\
\hline \multicolumn{6}{|l|}{ Others } \\
\hline Bird and flower market & 1 & 25 & 52 & 121.5 & 101.8 \\
\hline School & 1 & 6 & 67 & 73.8 & 33.9 \\
\hline Driving school & 2 & 7 & 37 & 38.8 & 25.5 \\
\hline Enterprise & 2 & 8 & 67 & 25.8 & 23.8 \\
\hline Park & 2 & 18 & 49 & 19.7 & 19.9 \\
\hline Hospital & 3 & 6 & 38 & 18.4 & 17.9 \\
\hline Hotel & 2 & 8 & 40 & 14.9 & 25.0 \\
\hline Subtotal & 13 & 78 & 48 & 30.6 & 30.1 \\
\hline Total & 60 & 166 & 49 & 11.3 & 16.1 \\
\hline
\end{tabular}

$\mathrm{CI}=$ container index, $\mathrm{RI}=$ route index, $\mathrm{TI}=$ time index .

\section{Results and Discussion}

The total inspection route length and elapsed time for the four study sites was $14.6 \mathrm{~km}$ and $10.3 \mathrm{~h}$, respectively (Table 1). Overall, 60 segments were inspected. The total number of potential mosquito habitats was 342, of which 166 were found to have mosquito larvae or pupae, and the total CI was 49\% (Table 2). Three mosquito species (Ae. albopictus, Culex quinquefasciatus Say, and Culex bitaeniorhynchus Giles) were collected and identified, of which Ae. albopictus was the most common species in small and large containers, stumps, other objects, plastic road barriers, potted or hydroponic plants, fallen leaves, and used tires. Large amounts of Ae. albopictus were found in one ditch. Larval Cx. quinquefasciatus mosquitoes were found in cisterns, puddles, storm drains, depressions of tree roots, ditches, and subterranean chambers. Cx. bitaeniorhynchus Giles was found just once in a ditch with moss. A few Ae. albopictus larvae were found with larval Cx. quinquefasciatus in underground cisterns, however, Culex molestus Forskal was not identified from the samples collected from the underground habitats [13]. Immature mosquitoes were collected from all standing-water 
habitats in underground parking lots [14]. No positive mosquito habitats were found in the farmer's market. Only two potential habitats $(\mathrm{CI}=50 \%)$ were found in the property management residential area. The CI for all habitats and premises ranged from $37 \%$ to $67 \%$, except for those with a CI of 0 or 100 . There was significant difference in the habitats $\left(X^{2}=27.994, \mathrm{df}=11, p<0.05\right)$ and no significant difference in the premises $\left(X^{2}=7.881, \mathrm{df}=10, p>0.05\right)$. The $\mathrm{CI}$ of underground habitats was significantly $(p<0.001)$ higher than those of the road, the residential area, and other habitats.

Twenty-five mosquito-positive habitats were found in the bird and flower market, which had an RI of 121.5, the highest among all studied premises types, followed by school areas $(\mathrm{RI}=73.8)$, and underground parking lots $(\mathrm{RI}=70.9)$ (Table 2$)$. The RI from the roadside was 4.2 , which was 5.3 times higher than the RI of grade C mosquito management criteria created by the NPHCC. The overall RI was 11.3 and the TI was 16.1. The TI in the bird and flower market was the highest ( $\mathrm{TI}=101.8)$, followed by the subdivision ( $\mathrm{TI}=35.3)$, and school (Table 2 ).

Small containers, stumps and other objects were the most abundant potential habitats (Figure 3), accounting for $26 \%(n=88), 12 \%(n=40)$, and $10 \%(n=35)$ of the total number, respectively. All 16 plastic road barriers and 6 used tires inspected presented larvae and/or pupae. None of the 19 shallow water habitats were mosquito positive. Immature mosquitoes were found in $66 \%$ of small containers, $50 \%$ of stumps, $46 \%$ of other objects, $35 \%$ of cisterns, $48 \%$ of puddles, $19 \%$ of storm drains, $29 \%$ of depressions of tree roots, $38 \%$ of potted or hydroponic plants and fallen leaves, $27 \%$ of ditches, $70 \%$ of large containers, and $33 \%$ of subterranean pits and chambers $\left(X^{2}=27.994 \mathrm{df}=11\right.$, $p<0.01)$. Immature mosquitoes were significantly more likely to be found in small containers than from other sources like, toys/shoes, cisterns, storm drains, tree roots, potted or hydroponic plants, and ditches $\left(\mathrm{X}^{2}=4.261, \mathrm{df}=1, p<0.05\right)$.

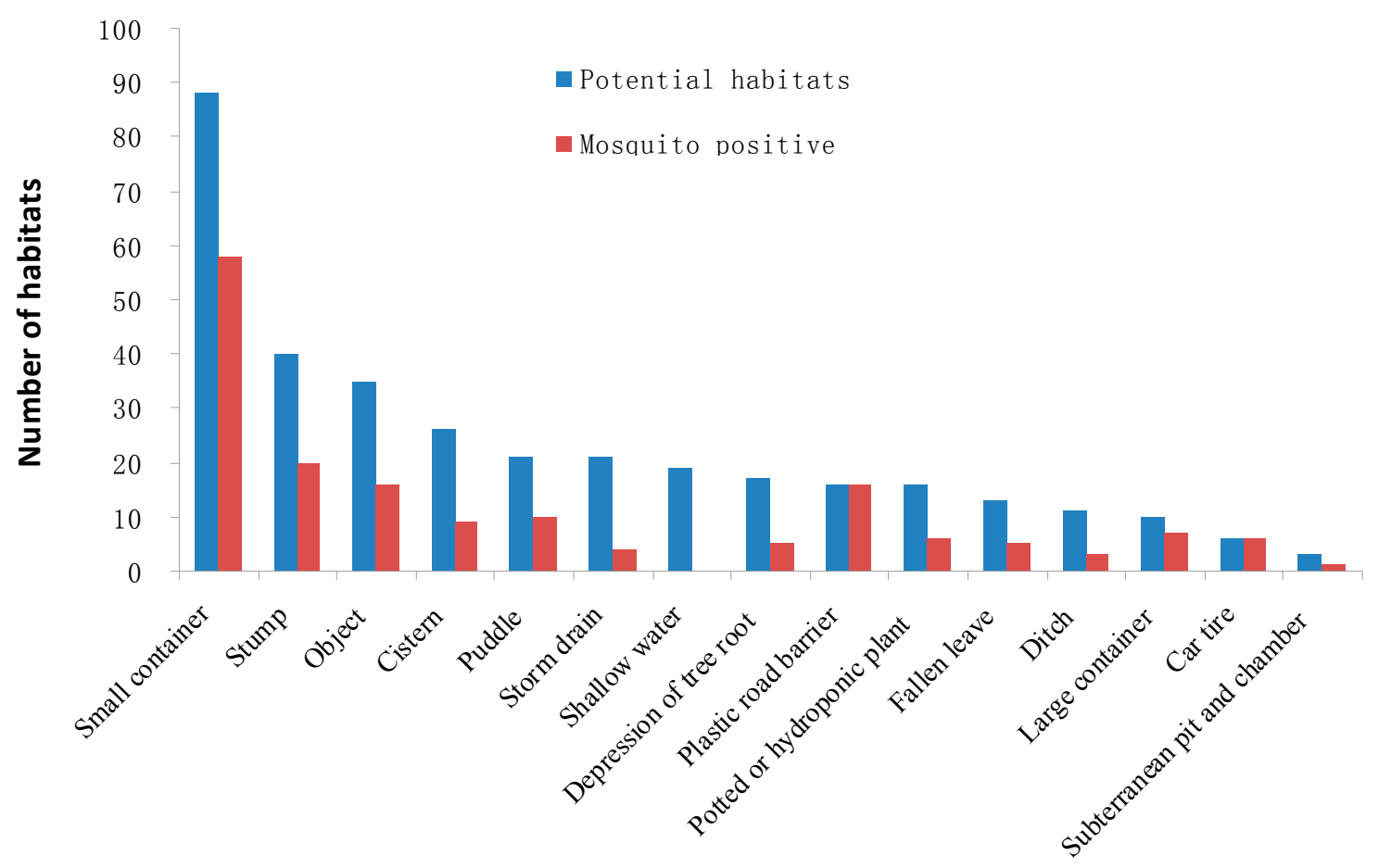

Figure 3. Numbers of potential habitats and mosquito-positive habitats by habitat type in Guangzhou.

Urban sources of Aedes albopictus are usually refractory to source-reduction in New Jersey, USA [15], however, the source reduction impacted the spatial distribution and abundance of the immature mosquitoes [6,11]. Based on our survey results, the source reduction effort should focus on small containers in Guangzhou City. To our knowledge, the Guangzhou government organized extensive "mosquito fogging" operations in the city to combat the dengue epidemic during mosquito 
activity seasons, and the larval source reduction responsibilities were given to the owner or manager of a premises. However, the general public lacked motivation, and thought this was the government's responsibility. The overall RI was 14.2 times more than the grade C criteria of the NPHCC. This suggests that larval source reduction, the key measure of fighting dengue $[11,16,17]$, was not sufficiently carried out in Guangzhou. We suggest that Guangzhou PHCC should promote integrated mosquito management and enhance source reduction for larval control during the adult control operation and urban vector management [2,18]. Since higher RI and/or TI values were in the bird and flower market, school, underground parking lot, and subdivisions, we suggest that the authorities should strengthen the surveillance on these premises types and focus on the mosquito management in wastelands, residential areas, underground structures, bird and flower markets, schools, and driving schools in Guangzhou.

During 2006-2012, Guangzhou Center for Disease Control and Prevention conducted routine Ae. albopictus larval surveillance in Guangzhou City using the Breteau index (BI) and CI [19]. The reported number of mosquito-positive habitats in Guangzhou City was underestimated, compared to our study results. This might be caused by inexperienced vector management professionals and different weather conditions. We suggest that the authorities enhance professional training and further study any possible impact factors on immature mosquito population and habitats.

The Android mobile device is a popular and convenient tool. An inspector could quickly gather all field information using these devices after training. Pictures, videos, geographic information of mosquito-positive habitats, and other data could be used for the proof and justification of the mosquito management responsibilities at the premises. Our study provides evidence that immature mosquito habitat surveillance in Guangzhou City, China, using Android mobile devices is feasible and convenient. This method provides much more useful data, compared with pen-and-paper-based field data collection. Lozano-Fuentes et al. (2013) [20] used a cell phone-based system (Chaak) to gather data during the surveillance of immature mosquitoes in the field. The data collected with cell phones was converted to the proper file format and finally it was transferred to a central electronic database. The Android mobile devices with OruxMaps used in our study collected field data more quickly than the cell phone Chaak app, as can be seen by comparing our report with that by Lozano-Fuentes et al. (2013). The application of Android mobile devices (and other new technology developed in the future) will be increased and benefit integrated mosquito management.

\section{Conclusions}

Immature mosquito habitats and the inspection routes were recorded by an Android mobile device with cameras and the OruxMaps and other apps in urban Guangzhou city, southern China, in 2015 after outbreaks of dengue fever in 2014. The total number of potential mosquito habitats was 342, of which 166 (49\%) were found with mosquito larvae or pupae. The overall route index (RI) was 11.3, which was 14.2 times higher than the grade C criteria of the National Patriotic Health Campaign Committee (NPHCC). The Android mobile device is a useful tool for surveillance of immature mosquito habitats.

Acknowledgments: We express our gratitude to Guo-Sheng Lian of Zhuhai International Travel Healthcare Center in Guangdong Province for field assistance. We also thank Hong Jiang of State Key Laboratory of Virology, College of Life Sciences, Wuhan University, China and Tong-Yan Zhao of State Key Laboratory of Pathogen and Biosecurity, Institute of Microbiology and Epidemiology, Beijing, China for their valuable suggestions, and Christopher Bibbs \& Daniel Dixon for language correction. Funding was provided by the Patriotic Health Campaign Committee Office of Wuhan.

Author Contributions: Tai-Ping Wu, Rui-De Xue, and Jun-Hua Tian contributed the experimental design. Tai-Ping $\mathrm{Wu}$, Yi-Liang Fang, Ai-Hua Zheng, and Jun-Hua Tian performed the experiment. Tai-Ping Wu and Jun-Hua Tian analyzed the data, and Rui-De Xue and Tai-Ping Wu wrote the manuscript. All authors read and approved the final manuscript.

Conflicts of Interest: The authors declare no conflict of interest. 


\section{References}

1. Gubler, D.J.; Reiter, P.; Ebi, K.L.; Yap, W.; Nasci, R.; Patz, J.A. Climate variability and change in the United States: Potential impacts on vector and Rodent-Borne Diseases. Environ. Health Perspect. 2001, 109, 223-233. [CrossRef] [PubMed]

2. Focks, D.A. A Review of Entomological Sampling Methods and Indicators for Dengue Vectors; World Health Organization: Geneva, Switzerland, 2003.

3. Song, S.F.; Luo, L.; Jin, Q.L.; Yang, Z.C. Epidemiological analysis of Dengue Fever in Guangzhou, 2001-2010. China Trop. Med. 2012, 12, 214-216. (In Chinese)

4. Li, B.; Morton, L.C.; Liu, Q.Y. Climate change and mosquito-borne diseases in China: A review. Glob. Health 2013. [CrossRef]

5. Kline, D.L. Mosquito population surveillance techniques. Tech. Bull. Fla. Mosq. Control Assoc. 2013, 7, 2-8.

6. Richards, S.L.; Ghosh, S.K.; Zeichner, B.C.; Apperson, C.S. Impact of source reduction on the spatial distribution of larve and pupae of Aedes albopictus (Diptera: Culicidae) in suburban neighborhoods of a Piedmont community in North Carolina. J. Med. Entomol. 2008, 45, 617-628. [CrossRef]

7. Weaver, J.H.R.; Phillips, J.D.; Gaines, M.K.; Xue, R.D.; Bequette, J.M. Analysis of Anastasia Mosquito Control District service requests. Wing Beats 2013, 24, 34-39.

8. Mark, T.; Wesley, S.; Yages, S.; Tanya, D.; Mickey, C.; Petrida, I.; Alexander, C.T.; Debra, J. The use of mobile phones as a data collection tool: A report from a household survey in South Africa. BMC Med. Inform. Decis. Mak. 2009. [CrossRef]

9. Zhang, J.; Li, C.S.; Li, X. Application of Oruxmaps software and android mobile on continuous forest inventory. Sichuan For. Explor. Des. 2012, 74-76, (Special volume in Chinese).

10. Dai, J.S.; Li, J.; Gu, Z.K.; Sun, B. A research on utility of pedometer to measure walking. J. Beijing Univ. Phys. Educ. 2008, 31, 219-222. (In Chinese)

11. Wu, T.P.; Wu, F.B.; Bao, J.Y.; Xu, X.T.; Huang, X.; Wang, H.; Liang, J.S.; Liu, G.X. Larval mosquito surveillance in mosquito management areas of Jiangan and Jianghan Districts of Wuhan. Chin. J. Vector Biol. Control 2004, 15, 467-468. (In Chinese)

12. Xing, W.Z. Application of the flat terminal transmission line geographic information and navigation tracking system. Autom. Appl. 2014, 11, 97-98. (In Chinese)

13. Wu, T.P.; Hu, Q.; Zhao, T.Y.; Tian, J.H.; Xue, R.D. Morphological studies of Culex molestus of Culex pipiens Complex (Diptera: Culicidae) in underground parking lots in Wuhan, Central China. Fla. Entomol. 2014, 97, 1191-1198. [CrossRef]

14. Wu, T.P.; Tian, J.H.; Zhao, T.Y.; Chen, X.M.; Xue, R.D. Mosquito Surveillance (Diptera: Culicidae) from Underground Parking Lots in Urban Area in Wuhan, Central China. Entomol. Ornithol. Herpetol. 2015. [CrossRef]

15. Unlu, I.; Farajollahi, A.; Strickman, D.; Fonseca, D.M. Crouching tiger, hidden trouble: Urban sources of Aedes albopictus (Diptera: Culicidae) refractory to source-reduction. PLoS ONE 2013, 8, e77999. [CrossRef] [PubMed]

16. Ooi, E.E.; Goh, K.T.; Gubler, D.J. Dengue prevention and 35 years of vector control in Singapore. Emerg. Infect Dis. 2006, 12, 887-893. [CrossRef] [PubMed]

17. Gubler, D.J. Dengue and Dengue Hemorrhagic Fever. Clin. Microbiol. Rev. 1998, 11, 480-496. [PubMed]

18. Wu, T.P.; Ma, L.H.; Liang, J.S.; Zhou, L.C.; Chen, X.M.; Liu, Q.; Bao, J.Y.; Tian, J.H. Vector management surveillance in Constructing National Sanitary City of Wuhan. Chin. J. Hyg. Insectic. Equip. 2015, 21, 451-457. (In Chinese)

19. Luo, L.; Li, X.N.; Xu, Y.; Huang, M.L.; Yang, Z.C. Identification of Aedes albopictus larval index thresholds in the transmission of Dengue in Guangzhou, China. J. Vector Ecol. 2015, 40, 240-246. [CrossRef] [PubMed]

20. Lozano-Fuentes, S.; Wedyan, F.; Hernandez-Garcia, E.; Sadhu, D.; Ghosh, S.; Bieman, J.M.; Tep-Chel, D.; Garcia-Rejon, J.E.; Eisen, L. Cell Phone-Based System (Chaak) for Surveillance of Immatures of Dengue Virus Mosquito Vectors. J. Med. Entomol. 2013, 50, 879-889. [CrossRef] [PubMed]

(C) 2016 by the authors; licensee MDPI, Basel, Switzerland. This article is an open access article distributed under the terms and conditions of the Creative Commons Attribution (CC-BY) license (http://creativecommons.org/licenses/by/4.0/). 